En collaboration avec le groupe de travail Qualité (GTQ) de la $\mathrm{FMH}^{*}$, le département Données, démographie et qualité (DDQ) élabore des documents de base sur divers thèmes ayant trait à la qualité, lesquels font l'objet d'une publication dans le Bulletin des médecins suisses. La FMH s'appuie sur les documents élaborés pour prendre position et faire connaître son point de vue au public au moyen du document intitulé «L'avis de la FMH». Le document de base et la prise de position de la FMH sur les erreurs de prescription sont présentés ci-dessous.

\title{
Erreurs de prescription
}

\author{
Varja A. Meyer-Nikolic, \\ Martina Hersperger, \\ Daniel Herren
}

\footnotetext{
* Membres du GTQ-FMH (par ordre alphabétique): Hélène Beutler,

Christoph Bosshard (depuis le 28 juin 2012), Karl Hampl, Catherine Heim, Daniel Herren (jusqu'au 7 juin 2012), Martina Hersperger, Stefanie Hostettler, Esther Kraft, Monika Loy, Francesca Mainieri, Varja Meyer Nikolic, Hans-Anton Vogel, Jürg von Below, Adrian Wirthner.
}

Correspondance:

Secrétariat général de la FMH

Département DDQ

Elfenstrasse 18

CH-3000 Berne 15

Tél. 0313591111

Fax 0313591112

ddq[at]fmh.ch

\section{Situation initiale}

On ne saurait concevoir le quotidien des médecins et des patients sans les médicaments. Or leur champ d'application thérapeutique n'est pas seulement vaste, il peut également avoir des conséquences néfastes importantes. Selon des études internationales parues à ce sujet, les erreurs médicamenteuses font partie des erreurs les plus fréquentes du secteur de la santé. Aux Etats-Unis, la littérature spécialisée estime que chaque année, $5,2 \%$ des patients hospitalisés sont touchés par des erreurs de prescription [1] et que dans le domaine ambulatoire, ce taux s'élève à un bon quart. Parmi celles-ci, 13\% sont graves et plus d'un tiers auraient pu être évitées ou atténuées [2]. Le nombre de problèmes évitables liés à la prescription de médicaments est estimé pour les seuls Etats-Unis à au moins 1,5 millions d'incidents par an [3]. Les patients sont certes les premiers concernés, mais les répercussions financières sont tout aussi importantes. Dans son rapport «Research on Patient safety» paru en 2008, l'OMS souligne à quel point il est important de promouvoir la recherche et d'intervenir dans les processus ayant trait à la sécurité des traitements médicamenteux [4].

Le caractère crucial de ces mesures pour la sécurité des patients a également été démontré en 2006 par une étude approfondie de la Fondation pour la sécurité des patients auprès de spécialistes hospitaliers [5]. Les études menées en Suisse estiment qu'au moins $7,5 \%$ de tous les patients hospitalisés sont sujets à une réaction indésirable de médicament et/ou sont concernés par une erreur de prescription. De plus, ces études montrent aussi qu'en Suisse, près de 7\% des hospitalisations sont dues à un problème associé à un médicament $[6,7,8]$. Les coûts directs qui en découlent sont estimés de 70 à 100 millions de francs [8]. Le taux élevé d'hospitalisations pour cause d'erreur médicamenteuse montre bien que la sécurité des médicaments n'est pas non plus optimale dans le domaine ambulatoire. Or, nous ne disposons pas en Suisse d'indications précises quant à la fréquence des erreurs de prescription pour le domaine ambulatoire. Comme pour le secteur hospitalier, la Fondation pour la sécurité des patients a également procédé à une enquête auprès des médecins du secteur ambulatoire

\section{Résumé}

Les erreurs médicamenteuses font partie des erreurs les plus fréquentes du domaine de la santé. Lors de la prise en charge médicamenteuse par les médecins, la prescription joue un rôle déterminant, car c'est justement dans cette première étape que les erreurs apparaissent le plus souvent, et ce tant dans le domaine ambulatoire qu'hospitalier.

Ce document a pour but de montrer la complexité du processus, les facteurs favorisant les erreurs et les mesures à prendre pour sécuriser les prescriptions.

et y a reconnu la nécessité d'agir [9]. Ces études sont corroborées par des études internationales montrant que les problèmes liés aux médicaments sont tout aussi fréquents dans le domaine ambulatoire, et que nombre d'entre elles auraient pu être évitées [10, 2].

Derrière l'objectif de fournir le bon médicament, au bon patient, au bon moment et selon le bon dosage, se trouve un processus complexe pouvant donner lieu à des erreurs. Ce processus englobe la prescription, la transcription, la dispensation, l'administration et la surveillance médicamenteuse. Chaque étape de ce processus caractérisé par l'implication de spécialistes d'horizons différents, a son propre potentiel d'erreur. Le présent document de base porte principalement sur la prescription de médicaments, autrement dit sur la partie du processus généralement accomplie par un médecin. Pour obtenir un aperçu détaillé des différentes étapes et des sources d'erreurs potentielles, nous recommandons la lecture des travaux l'Institute of Medicine of the National Academies [3] et de l'American Pharmacists Association [11].

\section{La prescription médicale: un sujet sensible}

La prescription de médicaments, acte essentiellement effectué par des médecins, joue un rôle crucial dans le processus médicamenteux, étant donné que celuici se fonde sur l'exactitude de la prescription. Or selon 
la littérature spécialisée, de nombreuses erreurs évitables ont justement lieu lors de cette première étape qui exige une prise de décision clinique, le choix du médicament approprié, la détermination du dosage adéquat, la prise en compte des caractéristiques du produit, l'inscription du processus dans le dossier médical et l'établissement d'une ordonnance orale ou écrite [3,12]. La figure 1 offre un aperçu du processus thérapeutique. Lors de la $1^{\text {re }}$ étape, les erreurs de prescription courantes concernent notamment la non-adaptation du dosage en cas de fonction rénale altérée, l'utilisation d'abréviations incorrectes, les lacunes dans la transcription ou la non-prise en compte des indications documentées du patient, notamment en cas d'allergie.

Même si les erreurs de prescription ne causent pas toutes un dommage à la santé, leurs conséquences sont à prendre au sérieux. Elles peuvent en effet entraîner des interactions indésirables, voire dangereuses, occasionner des surdoses ou des réactions allergiques et même, en cas de dosage trop faible, faire échouer le traitement. A cela s'ajoutent l'absence de

Décision clinique à la base du traitement médicamenteux

Diagnostic correct, patient correct, diagnostic secondaire, capacité d'observance médicamenteuse, accord du patient

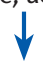

Choix du médicament approprié

Alternatives au traitement, diagnostic secondaire, allergies, rapport coûts/utilité, modalités d'administration

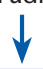

Détermination du dosage

Age/poids/grandeur, diagnostic, sévérité de la maladie, diagnostic secondaire, traitement complémentaire

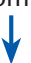

Prise en compte des caractéristiques du produit

Dosage, rythme de l'administration, interactions médicamenteuses, date de péremption

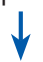

Documentation de la médication

Anamnèse, communication avec d'autres personnes en charge du traitement, documentation pour le patient

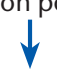

\section{Prescription écrite}

Etablissement de I'ordonnance, lisibilité, ordonnance correcte (vérification)

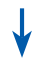

Instructions au patient

Communication adaptée (langue, lisibilité), documentation écrite complémentaire (notice, instructions personnelles du médecin)

Figure 1

Etapes du processus de prescription médicamenteuse (inspiré du schéma du «Comittee on Identifying and Preventing Medication Errors» de 2007 avec quelques précisions). prescriptions malgré l'indication et les prescriptions malgré l'absence d'indications, deux situations pouvant elles aussi occasionner des effets indésirables [13]. Une récente étude suisse sur les interactions de médicaments montre par ex. que $27 \%$ des prescriptions hospitalières provoquent des interactions entre les médicaments et que pour $5 \%$ des cas, ces prescriptions sont clairement contre-indiquées [14].

\section{Facteurs entraînant des erreurs de prescription}

En pratique, la prescription de médicaments est aussi complexe que l'analyse des erreurs car dans la plupart des cas, ce ne sont pas des facteurs isolés qui les occasionnent, mais la combinaison de plusieurs facteurs ou situations à risque $[15,16]$.

Différentes études, principalement dans le secteur hospitalier, en ont analysé les causes [15, 17, 16]. Des entretiens qualitatifs avec des médecins anglais impliqués dans des erreurs de prescription ont montré que les principales causes étaient liées à l'environnement professionnel (charge de travail élevée, environnement professionnel et effectifs), à l'équipe médicale (malentendus ou défaut de communication, responsabilités mal définies, manque de supervision chez les médecins-assistants), à des facteurs personnels (mal-être physique ou psychique, fatigue ou stress, manque de connaissances) [15, 18] (fig. 2).

Une autre source d'erreurs importante dans les prescriptions manuscrites est due au manque de lisibilité et aux difficultés de transmission. Seules $2 \%$ des prescriptions manuscrites analysées ont été jugées lisibles [17]. Les ordonnances illisibles ou incompréhensibles peuvent avoir des conséquences médicales graves ou conduire à des interruptions de travail inutiles nécessitant de prendre contact avec le médecin prescripteur. De telles interruptions des flux de travail peuvent potentiellement entraîner de nouvelles erreurs [19]. De plus, si l'établissement concerné a une culture d'entreprise qui n'offre pas ou difficilement la possibilité aux médecins-assistants ou aux soignants de poser des questions à leurs supérieurs, la situation s'aggrave encore plus [15].

Les études explicites portant précisément sur les causes à l'origine des erreurs de prescription sont rares dans le domaine ambulatoire et la raison en est probablement la difficulté plus grande d'y récolter des données que dans le secteur hospitalier. On peut néanmoins partir du principe que les facteurs d'erreurs sont identiques dans les deux secteurs. La difficulté principale dans le secteur ambulatoire concerne la gestion des ordonnances, étant donné que ce sont souvent des médecins différents qui prescrivent des médicaments à un même patient [20]. Des dossiers de patient incomplets et un manque de communication entre les différents spécialistes impliqués dans le traitement ou dans la relation avec le patient sont aussi une cause d'erreurs importante [3, 11]. Le manque de connaissance des spécificités du patient, telle que grossesse, allergies, données de laboratoire, 


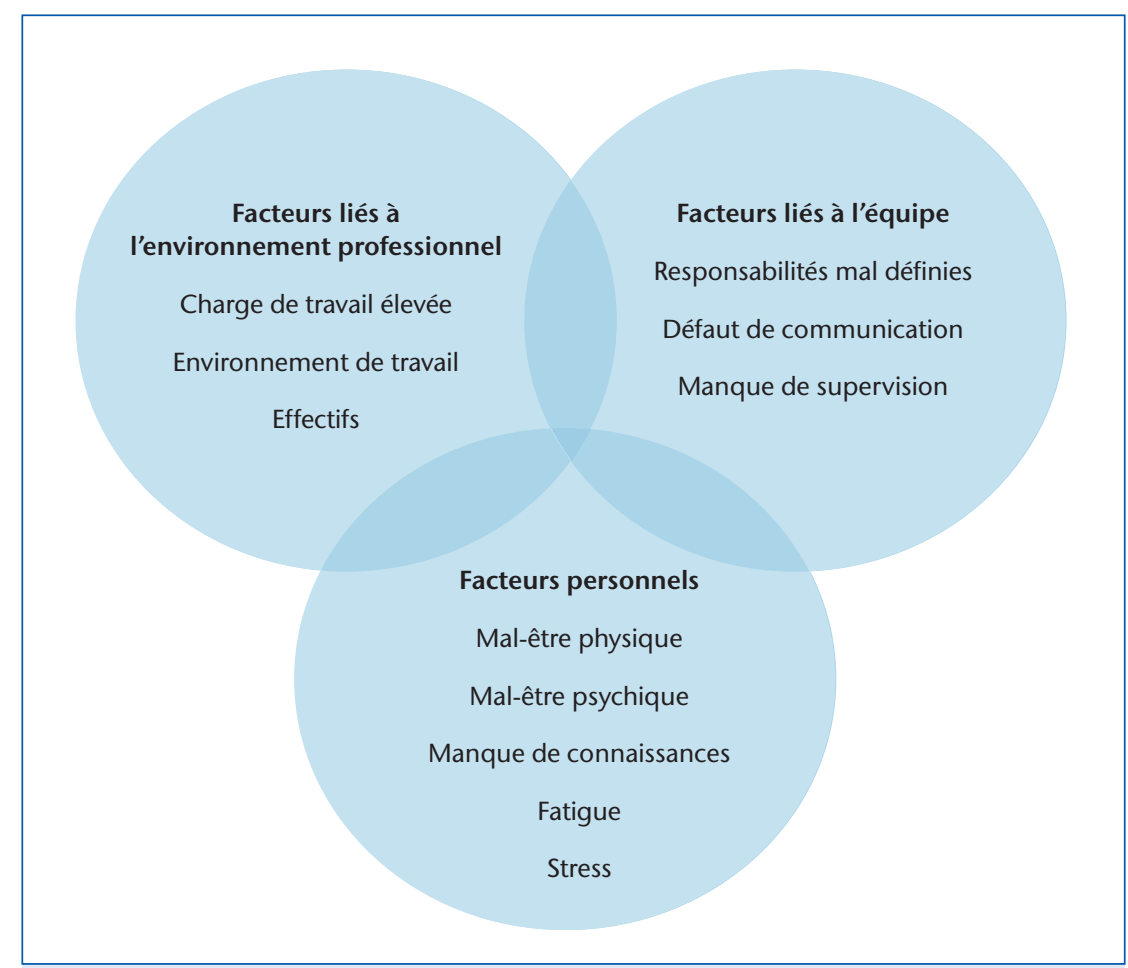

Figure 2

Facteurs favorisant les erreurs de prescription dans le domaine hospitalier du point de vue du médecin prescripteur (inspiré de Dean B. et al. (2002)).

âge et poids, diagnostic, médicaments en automédication ou prise de compléments alimentaires représente également un risque pour la sécurité des prescriptions de médicaments dans cette catégorie [3, 11].

En règle générale, on peut relever que la communication joue un rôle crucial dans la sécurité des prescriptions. Communiquer sans malentendu est non seulement essentiel au sein de l'équipe de traitement, mais aussi dans la relation avec le patient et en cas de recoupement thérapeutique lors de l'admission ou de la sortie d'hôpital par ex. Ces interfaces sont délicates et exigent d'accorder une attention particulière au flux d'informations et au travail en réseau [21].

Une autre cause d'erreur déterminante est le manque de conscience du degré élevé d'erreurs potentielles inhérentes au processus de prescription [16, 15]. A cela s'ajoute qu'une approche ouverte et constructive des erreurs de prescriptions - à l'instar de ce qui se fait dans les systèmes d'annonce d'incidents critiques - n'est de loin pas une évidence, ce qui empêche l'analyse des erreurs commises et entraîne leur répétition.

\section{Mesures à prendre pour assurer la sécurité des prescriptions}

La compréhension des causes très diverses des erreurs de prescription contribue à les prévenir et à rechercher des solutions pour les éviter [15]. Il importe tout d'abord de se rendre mieux compte que la prescription de médicaments est un acte à haut risque. La vérification des processus de prescription et l'analyse des erreurs dans ce domaine grâce à une culture des erreurs bien implantée fait partie des solutions préconisées. Il est essentiel d'accorder à ces mesures l'attention qu'elles méritent [15].

$\mathrm{Si}$, au départ, il faut définir le bon médicament et le bon dosage, il est ensuite nécessaire de garantir l'accès aux documents de référence tels que les guides de pratique ou les informations sur les médicaments [16]. Dans le domaine hospitalier, une mesure de sécurité supplémentaire consiste également à demander l'avis d'un pharmacien [15]. Par ailleurs, l'on dispose aujourd'hui de différents outils de prescription informatisés. Certaines études ont montré que ces méthodes permettaient de réduire considérablement le nombre d'erreurs [22], alors que d'autres ont souligné qu'elles risquaient de provoquer de nouvelles erreurs si l'on ne sait pas bien les utiliser [23]. De plus, de nombreux outils de prescription informatisés ne sont pas suffisamment adaptés aux spécificités des patients, ce qui peut notamment entraîner des signaux faussement positifs auxquels on accordera par erreur aucune importance dans une situation pourtant complexe pour le patient [24].

Si le bon médicament a été prescrit selon le bon dosage, il est nécessaire de prendre des mesures pour que la prescription soit correctement communiquée. Il convient donc de renoncer aux prescriptions orales, car elles peuvent être source de malentendus. Le cotrimazol peut ainsi aisément se transformer en clotrimazol et vice-versa [19]. Mais les ordonnances écrites ne sont pas non plus irréprochables, car même les mesures de précaution simples telles qu'une écriture manuscrite lisible ou des règles d'abréviation correctes ne sont pas toujours respectées. Cohen (2007) fournit un bon aperçu de cette problématique [19]. La documentation électronique offre une contribution importante, en particulier les dossiers médicaux informatisés qui garantissent une bonne lisibilité [25].

Cependant, aucune mesure citée ne permettra d'empêcher ou d'éviter toutes les erreurs de prescription. Il est donc d'autant plus important d'aborder cette thématique selon différentes perspectives. La Commission on Safety and Quality in Health Care en Australie [26] ou le Bundesministerium für Gesundheit en Allemagne ont par exemple défini des approches globales [27]. Depuis 2007, l'Allemagne met sur pied un plan d'action détaillé pour améliorer la sécurité des prescriptions médicamenteuses et pour garantir une plus grande sécurité dans l'ensemble du processus. Parmi les démarches prévues, on trouve également des mesures concrètes visant à optimiser la sécurité des prescriptions [27].

\section{Situation en Suisse}

En Suisse, nous sommes encore loin de la stratégie à large échelle appliquée en Allemagne. Cependant, le taux élevé de problèmes dus à des erreurs médicamenteuses marque également notre politique de santé. Outre les diverses interpellations récentes au 
Conseil national et au Conseil des Etats [28] visant à améliorer la sécurité des prescriptions, la stratégie fédérale en matière de qualité dans le système de santé et le rapport sur la concrétisation de cette stratégie mettent également la priorité sur cette question. Les rapports à ce sujet proposent des mesures telles que l'ordonnance électronique, les services de consultation pharmaceutique axés sur le réexamen personnalisé de la médication et les conseils en matière de médicaments à la sortie de l'hôpital [29, 30]. L'ordonnance électronique est également l'un des deux axes prioritaires lors de la concrétisation de la stratégie nationale en matière de cybersanté [33].

Outre ces mesures de politique de santé, des initiatives individuelles prometteuses ont également été mises en œuvre. La Fondation pour la sécurité des patients s'engage ainsi depuis longtemps dans divers projets visant à garantir la sécurité des traitements médicamenteux [31]. Les cercles de qualité entre médecins et pharmaciens sont un autre exemple d'amélioration permanente qui permet de plus de baisser les coûts sans nuire à la relation médecin-patient et pharmacien-patient [32].

\section{Conclusions}

La prescription de médicaments est certes un processus complexe et difficile, mais la fréquence des erreurs de prescription est beaucoup trop élevée. Une mesure unique pour améliorer la sécurité des prescriptions ne suffira pas. Il est urgent et nécessaire d'opter pour une approche pluridimensionnelle. Celle-ci devra comprendre la promotion de la recherche pour développer et implanter des outils pratiques ainsi que la coordination des différentes approches sans oublier l'application de mesures ayant déjà fait leurs preuves. Dans le domaine ambulatoire en particulier, il serait important de mandater de nouvelles études pour déterminer les causes des erreurs de prescription.

Puisque l'ensemble du processus médicamenteux se base sur une prescription correcte, il est essentiel d'analyser cette première étape du processus à long terme. L'implication des médecins est donc fortement encouragée puisque la prescription de médicaments relève principalement d'eux. Etant donné toutefois que les causes d'erreurs sont souvent liées au système de prescription, il est impératif de chercher une solution commune impliquant tous les groupes professionnels et institutions concernés.

Etant donné la complexité du sujet, il est impossible de déterminer toutes les causes d'erreurs susceptibles d'être corrigées. Le présent document ne prétend donc pas à l'exhaustivité, mais entend sensibiliser aux risques élevés d'erreurs dans le domaine des prescriptions de médicaments.

\section{Références}

1 Bond CA, Raehl CL, Franke T. Clinical Pharmacy Services, Hospital Pharmacy Staffing, and Medication
Errors in the United States Hospitals. Pharmacotherapy. 2002;22(2):134-47.

2 Gandhi TK, Weingart SN, Borus J, Seger AC, Peterson J, et al. Adverse Drug Events in Ambulatory Care. The New England Journal of Medicine. 2003;248(16): 1556-64.

3 Comittee on Identifying and Preventing Medication Errors, Institute of Medicine of the National Academies. Preventing Medication Errors. Washington: The National Academies Press; 2007.

4 World Health Organisation WHO/The Research Priority Setting Working Group of the World Alliance for Patient Safety. Summary of the evidence on patient safety: Implications for Research. Lien internet: http://whqlibdoc.who.int/publications/2008/ 9789241596541_eng.pdf; 2008. [Etat au 17.2.2012].

5 Frank O, Hochreutener MA. Problemfelder (Hot-Spots) in der Patientensicherheit. Schweizerische Ärztezeitung. 2008; 89(24):1079-82.

6 Hardmeier B, Braunschweig S, Cavallaro M, Roos M, Pauli-Magnus, et al. Adverse drug events caused by medication errors in medical inpatients. SwissMedWeekly. 2004;134(45-46):664-70.

7 Fattinger K, Roos M, Vergères P, Holenstein C, Kind B, et al. Epidemiology of drug exposure and adverse drug reactions in two swiss departments of internal medicine. British Journal of Clinical Pharmacology. 2000;49(2):158-67.

8 Lepori V, Perren A, Marone C. Adverse internal medicine drug effects at hospital admission. Schweizerische Medizinische Wochenschrift. 1999;129(24):915-22.

9 Gehrin K, Schwappach D. Patientensicherheit in der Grundversorgung - erste Daten aus der Schweiz. Schweiz Ärztezeitung. 2011;92(39):1486-7.

10 Aagaard Thomsen, L, Winterstein AG, Søndergaard B, Stig Haugbølle L, Melander A. Systematic Review oft he Incidence and Characteristics of Preventable Adverse Drug Events in Ambulatory Care. The Annals of Pharmacotherapy. 2007; Sept.,Vol. 41:1411-26.

11 Cohen M. Causes of Medication Errors. In: Cohen M (eds.). Medication Errors. Washington: American Pharmacists Association; 2007. p. 55-66.

12 Bates DW, Cullen DJ, Laird N, Petersen LA, Small SD et al. Incidence of adverse drug events and potential adverse drug events: implications for prevention. JAMA. 1995;274:29-34.

13 Wessell AM, Litvin C, Jenkins RG, Nietert PJ, Nemeth LS, et al. Medication prescribing and monitoring errors in primary care: a report from the Practice Partner Research Network. Quality and Safety in Health Care BMJ. 2010;19(e21). Online in Internet: http://qualitysafety.bmj.com/content/19/5/1.39.short [18.10.2012].

14 Oertle M. Frequency and nature of drug-drug interactions in a Swiss primary and secondary acute care hospital. SwissMedWeekly. 2012;142:w13522.

15 Dean B, Schachter M, Vincent C, Barber N. Causes of prescribing errors in hospital inpatients: a prospective study. The Lancet. 2002; Vol. 359, April 20:1373-8.

16 Coombes ID, Stowasser DA, Coombes JA, Mitchell C. Why do interns make prescribing errors? A qualitative study. Medical Journal of Australia. 2008;188(2):89-94.

17 Hartel M, Staub LP, Röder C, Eggli S. High incidence of medication documentation errors in a Swiss university 
hospital due to the handwritten prescription process. BMC Health Services Research. 2011;11:199.

18 Le département Données, démographie et qualité (DDQ) de la FMH a publié son propre document de base sur le rapport entre bien-être du médecin et qualité des traitements: Hostettler, Hersperger, Herren. Le bien-être du médecin influe sur la qualité du traitement. Bull Méd Suisses. 2012;93(18):655-9.

19 Cohen M. Causes of Medication Errors. In: Cohen M. (eds.). Medication Errors. Washington: American Pharmacists Association; 2007. p. 175-203.

20 Rundall TG, Hsu J, Elston Lafata J, Fung V, Paez KA, et al. Prescribing Safety in Ambulatory Care: Physician Perspectives. In: Henriksen K, et al. (eds.). Advances in Patient Safety: From Research to Implementation (Vol. 1: Research Findings). Rockville (MD): Agency for Healthcare Research and Quality (US); 2005. p. 161-71.

21 Hersberger K, Petitjean-Wiesner C. Arzneimittelversorgung in der Perihospitalphase: Die Schnittstelle Spital/ Apotheke/Hausarzt. Managed Care. 2004;1:10-2.

22 Cf. par ex. Kaushal R, Kern LM, Barrón Y, Quaresimo J, Abramson EL. Electronic Prescribing Improves Medication Safety in Community-Based Office Practices. Journal of General Internal Medicine. 2010;25(6): 530-6.

23 Cf. par ex. Redwood S, Rajakumar A, Hodson J, Coleman JJ. Does the implementation of an electronic prescribing system create unintended medication errors? A study of the sociotechnical context through the analysis of reported medication incidents. BMC Medical Informatics and Decision Making. 2011;11:29. Lien internet: www.biomedcentral.com/1472-6947/ 11/29 [Etat au 22.2.2012].

24 Taylor LK, Kawasumi Y, Bartlett G, Tamblyn R. Inappropriate Prescribing Practices: The Challenge and Opportunity for Patient Safety. Healthcare Quarterly. 2005; Vol. 8, Special Issue:81-5.
25 Schade CP, Sullivan FM, De Lusignan S, Madeley J. e-Prescribing, Efficiency, Quality: Lessons from Computerization of UK Familiy Practice. Journal of the American Medical Informatics Association. 2006;13:470-5.

26 Australian Commission on Safety and Quality in Health Care: Medication Safety. Lien internet: www.safetyandquality.gov.au/our-work/medicationsafety/ [18.10.2012].

27 Bundesministerium für Gesundheit: Aktionsplan zur Verbesserung der Arzneimitteltherapiesicherheit in Deutschland. Lien internet: www.ap-amts.de/ [Etat au 17.2.2012]

28 Cf. par ex. www.parlament.ch/d/suche/seiten/ geschaefte.aspx?gesch_id=20103093 [Stand 6.3.2012].

29 Office fédéral de la santé publique. Stratégie en matière de qualité dans le système de santé suisse. Lien internet: www.bag.admin.ch/themen/krankenversicherung/ 00300/00304/index.html?lang=de; 2009. [Etat au 23.11.2011].

30 Office fédéral de la santé publique. Rapport au Conseil fédéral sur la concrétisation de la stratégie fédérale en matière de qualité dans le système de santé. Lien internet: www.bag.admin.ch/themen/ krankenversicherung/00300/00304/index. html?lang=de; 2011. [Etat au 23.11.2011].

31 Cf. www.patientensicherheit.ch. [Etat au 17.2.2012].

32 Nyffeler R. Qualitätszirkel zur Medikamentenverschreibung - eine Antwort auf die Kostenentwicklung im Gesundheitswesen. Managed Care. 2002;(2):23-6

33 Cf. le site de eHealth Suisse: www.e-health-suisse.ch/ umsetzung/00146/00148/index.html?lang=de [Etat au 4.7.2012]. 